\title{
Bilateral hilar adenopathy: an unusual presentation of renal cell carcinoma
}

\author{
TE KING JR, J FISHER, MI SCHWARZ, LH PATZELT
}

\begin{abstract}
From the Division of Pulmonary Medicine, Department of Medicine, and Department of Surgery, The University of Colorado Health Sciences Center and Department of Medicine, Denver Veterans Administration Medical Center, Denver, Colorado, USA
\end{abstract}

Isolated symmetrical hilar adenopathy in an asymptomatic patient is an unusual presentation for pulmonary metastatic disease. In a large series of patients with bilateral hilar adenopathy only those with sarcoidosis were free of symptoms. In contrast, all the patients with hilar adenopathy secondary to extrathoracic neoplasms were symptomatic and the primary tumour was apparent. $^{1}$ The purpose of this communication is to describe an asymptomatic patient in whom symmetrical hilar adenopathy developed as an isolated, late manifestation of renal cell carcinoma.

\section{Case report}

A 49-year-old man was evaluated in January 1978 for haematuria. An intravenous pyelogram revealed a right renal mass which proved to be a carcinoma on renal angiography. A right radical nephrectomy was performed. The preoperative chest radiograph was normal. Pathological examination showed renal cell carcinoma without extension to vessels or to the capsule, though tumour was present in one perirenal lymph node. The

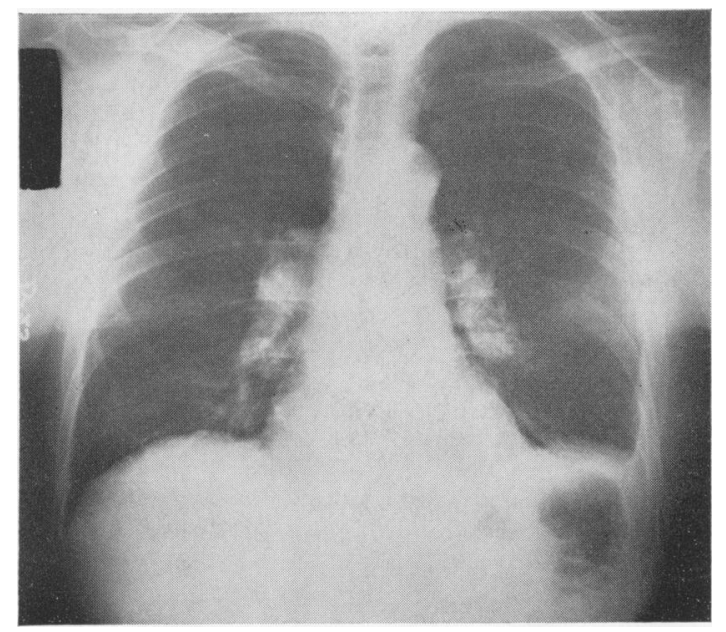

Fig 1 Posteroanterior chest radiograph films demonstrating bilateral hilar adenopathy, fractured left eighth rib and small left pleural effusion.

Address for reprint requests: Dr TE King, Pulmonary Service, Veterans Administration Medical Center, 1055 Clermont Street, Denver, Colorado 80220, USA. patient received 4800 rads to the renal pedicle over a fiveweek period.

In July 1980, the patient sustained a rib fracture after being thrown from a horse. The chest radiograph revealed bilateral hilar adenopathy as well as a benign rib fracture and small associated pleural effusion (fig 1). Computerised tomography of the chest showed no intrapulmonary lesions. The patient underwent mediastinoscopy and left thoracotomy. A biospy of the hilar lymph nodes showed histological changes consistent with metastatic renal cell carcinoma (fig 2).

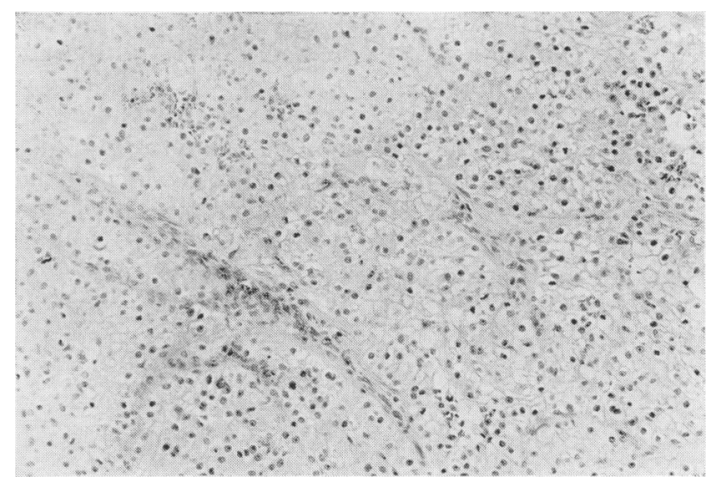

Fig 2 Photomicrograph of the hilar node biopsy, demonstrating clear cells with abnormal nuclei. Haematoxylin and eosin, $\times 160$.

\section{Discussion}

Bilateral pulmonary hilar adenopathy in an asymptomatic patient is characteristic of sarcoidosis. Lymphoma, tuberculosis, and fungal infections, berylliosis, and bronchogenic carcinoma are other unusual causes of hilar adenopathy but are extremely rare in an asymptomatic patient. Extrathoracic neoplasms are infrequently associated with this finding. Those which metastasise to intrathoracic lymph nodes include tumours of the head and neck, genitourinary tracts (primarily testicular tumours), breast, and malignant melanomas. ${ }^{12}$ When intrathoracic lymphadenopathy results from metastatic disease the patient is usually symptomatic and the lymphadenopathy is usually unilateral and associated with other intrathoracic abnormalities. Moreover there is usually an identifiable primary extrathoracic tumour site. $^{2}$ 
Renal cell carcinoma because of its tendency to invade lymph and blood vessels can present with a variety of clinical and radiographic manifestations. ${ }^{3}$ Thoracic involvement occurs in $55 \%$ of patients. ${ }^{3}$ The most common form of metastasis to the thorax is multiple pulmonary nodules, usually in the form of large ("cannonball") pulmonary masses. Lymphangitic carcinomatosis, pleural involvement with or without effusion, endobronchial obstruction, tumour emboli, and bony thoracic cage involvement are also seen. Hilar or mediastinal nodal involvement or both have been reported but almost invariably occurs in conjunction with one of the other thoracic manifestations noted above..$^{3-8}$ In addition, when hilar adenopathy occurs the primary neoplasm is usually large $(>6 \mathrm{~cm})$ and has penetrated the renal capsule with extension into the perinephric fat and vascular structures of adjacent tissue. ${ }^{4}$

Renal cell carcinoma reaches the thorax by either invasion of veins or retroperitoneal lymphatics with dissemination via the thoracic duct and reflux into the bronchomediastinal lymphatic trunks. The latter mechanism would most likely account for the development of bilateral hilar lymph node involvement and is supported by the finding that $5-10 \%$ of patients undergoing pedal lymphangiography have reflux of contrast material into the hilar lymphatics, bronchomediastinal trunks, and peribronchial lymphatics. ${ }^{4} 9$

Our patient's primary tumour was diagnosed 30 months before the development of asymptomatic hilar nodes. The original tumour was relatively small and had not penetrated the renal capsule; therefore, it was not typical of those tumours likely to metastasise to the hilar lymph nodes. In addition, surgical excision and radio- therapy were performed without evidence of regional recurrence. Such development of late metastatic disease even after apparently curative local therapy, is well recognised in renal cell carcinoma. ${ }^{10}$

\section{References}

${ }^{1}$ Winterbauer RH, Belic N, Moores KD. A clinical interpretation of bilateral hilar adenopathy. Ann Intern Med 1973;78:65-71.

${ }^{2}$ McLoud TC, Kalisher L, Stark P, Greene R. Intrathoracic lymph node metastases from extrathoracic neoplasms. Am J Roentgenol 1978;131:403-7.

${ }^{3}$ Latour A, Shulman HS. Thoracic manifestations of renal cell carcinoma. Radiology 19760;21:43-8.

${ }^{4}$ Lang EK. Renal cell carcinoma presenting with metastases to pulmonary hilar nodes. J Urol 1977;118:543-6.

${ }^{5}$ Arkless R. Renal carcinoma: how it metastasizes. Radiology 1965 ;84:496-501.

${ }^{6}$ Reinke RT, Higgins CB, Niwayama G, Harris RH, Friedman PJ. Bilateral pulmonary hilar lymphadenopathy. An unusual manifestation of renal cell carcinoma. Radiology 1976;121:49-53.

${ }^{7}$ Khan A, Khan FA. Hypernephroma: a rare cause of bilateral adenopathy, and an example of the importance of tissue diagnosis in suspected cases of sarcoidosis. Chest 1974;66:722-3.

${ }^{8}$ Wright FW. Enlarged hilar and mediastinal nodes (and especially lower right hilar node enlargement) as a sign of metastasis of renal tumor. Clin Radiol 1977;28: 431-6.

${ }^{2}$ Weidner WA, Steiner RM. Roentgenographic demonstration of intrapulmonary and pleural lymphatics during lymphangiography. Radiology 1971 ;100:533-9.

10 Berman LB, Giardina ACV, Estes D. Hilar enlargement twenty years following resection of a renal cell tumor. Mount Sinai J Med 1979;46:378-81. 